\title{
The risk of forcing inbreeding in conservation programmes: a reply to Theodorou and Couvet
}

\author{
Heredity (2017) 119, 51-53; doi:10.1038/hdy.2017.17; published online 22 March 2017
}

The possibility of applying different degrees of forced inbreeding in conservation programmes has been suggested, on a number of occasions, as a way for purging part of the deleterious mutation load (eg, Templeton and Read, 1984). One of the latest proposals in this respect was made by Theodorou and Couvet (2015) (T\&C15 hereafter), who showed by computer simulations the possible benefits of applying circular sib mating $(\mathrm{CM})$ in conservation programmes. However, simulations performed by Caballero et al. (2016) using the number of lethal equivalents empirically observed in wild populations ( $B=6$; O'Grady et al., 2006) suggested that CM is a too-risky strategy to be advised in conservation programmes given its attached high probability of line extinction.

Theodorou and Couvet (2016) (T\&C16 thereafter) have recently argued that the conclusion reached by Caballero et al. (2016) is not justified because the assumed inbreeding load, $B=6$, can be significantly higher than that in captive conditions. The reasons given are basically three: (1) There is a substantial relaxation of selection in benign, captive conditions relative to stressful wild ones. (2) Populations under a conservation programme may have passed through previous purging because of a history of size decline or bottlenecking; (3) A part of inbreeding depression is due to the expression of traits that are marginally relevant to populations in captivity. An additional point considered by $\mathrm{T} \& \mathrm{C} 16$ refers to the appropriateness of the mutational parameters assumed by Caballero et al. (2016).

Although the above arguments are all reasonable discussion points, in our opinion, the risks associated with a programme involving forced inbreeding are still too high, and hence such a design should not be advised for application in conservation programmes. Regarding the first point, it is reasonable to assume that the deleterious effects of mutations are likely to be lower in the benign captive conditions, and this is supported by the meta-analysis of Fox and Reed (2011), who showed that inbreeding depression increases with environmental stress. Caballero et al. (2016) considered this factor by halving the selection coefficients of deleterious mutations in their simulated captive populations, and thereby halving the inbreeding load from $B=6$ in natural conditions to $B=3$ in captive conditions. This reduction by a half of the selection coefficients was also assumed by T\&C15 when presenting their simulations for the probability of extinction during captive breeding (see their Table 1). However, $\mathrm{T} \& \mathrm{C} 16$ suggest that this reduction might not be enough and provide some references to support the argument. Fox and Reed (2011) deduced that environmental stress would produce additional lethal equivalents. In particular, they reckoned that one lethal equivalent would be generated, on average, for each 30\% reduction in fitness induced by stressful conditions. Therefore, the assumption made by Caballero et al. (2016) that the number of lethal equivalents is reduced from six in natural to three in captive conditions would imply a $90 \%$ reduction in fitness due to environmental stress in natural conditions, which seems to be a substantial effect. In addition, a recent comparison of inbreeding loads in competitive versus noncompetitive conditions in Drosophila suggests that the former is about twice as large as the latter (López-Cortegano et al., 2016), supporting the above mentioned assumption of halving the selection coefficient in captive conditions. Nevertheless, the consideration of any extent of relaxed selection in captive conditions is somehow arbitrary, as it is not really known. A large meta-analysis on the impact of environmental factors on mutational effects carried out by Martin and Lenormand (2006) shows that although the mutational variance of fitness effects tends to be larger in adverse environments than in benign ones, the average change in their mean is not. This casts doubts on the uniform reduction in selection coefficients in benign environments. Although all deleterious mutations become uniformly much less harmful in benign captive conditions, CM does not necessarily become favourable because its purging effect would be much compromised.

With respect to the second point, it is reasonable to assume that populations under threat may have previously passed through severe historical bottlenecks, so that purging has already taken place before a conservation programme starts. This is a realistic assumption not considered by either T\&C15 or Caballero et al. (2016). But two points should be noted here, one is that besides a reduction in inbreeding load because of previous purging, a history of small population sizes also may incur fixation of deleterious mutations affecting all fitness components, implying that the reproductive capability of the population will be impaired at the start of the conservation programme. This will point towards a disadvantage of CM relative to methods avoiding inbreeding, such as equalization of contributions (EC), because the lower the reproductive capability of the population the higher the risks of extinction under forced inbreeding. The other and more important point is that, if the population has suffered from a previous history of reduced population size, a substantial part of the inbreeding load may have been lost, and the possible benefits of $\mathrm{CM}$ in removing such a load may partially vanish. In this scenario, it is expected that mutations purged from bottlenecked populations in natural conditions will be those of large deleterious effects, while those remaining in captive populations will be of small deleterious effects that are difficult or impossible to purge. Therefore, it is unlikely that CM will have an advantage under a scenario of strong previous purging.

In order to illustrate this latter point, we performed simulations under a scenario where the large-base population passes through a period of reduced population size prior to the start of the conservation programme. In particular, we considered a large population with 
effective size $N_{e}=1000$ over 10000 generations under the mutational parameters of model A in Caballero et al. (2016), which would imply an inbreeding load of about $B=6$ in the population. However, now a reduction of effective population size (to $N_{e}=10$ ) was assumed in the last 20 generations before starting the conservation programme. To avoid possible extinctions of these bottlenecked populations, no limitation in the reproductive capacity of individuals was assumed for this period of reduced population size. The result of this continued bottlenecking was a substantial purging of the inbreeding load in the population, from $B=6$ down to 2.19. Assuming this initial scenario, captive breeding programmes were set up assuming CM or EC under a range of population sizes (10 to 100). Because selection coefficients were again assumed to be halved in captivity, the actual expected inbreeding depression at the start of the conservation programme would correspond to $B=1.2$, a value substantially lower than that assumed for wild populations $(B=6)$.

Figure 1 shows the probability of extinction under both conservation strategies (CM and EC) after 10 and 25 generations for situations with different maximum captive population sizes $\left(N_{\max }\right)$ and a reproductive rate of $K=1.5$ (an average maximum number of three progeny per individual at the start of the conservation programme). The results should be compared with those in Figure 5 of Caballero et al. (2016). It is seen that, even for such a previously reduced inbreeding load ( $B$ down to 1.2 ), the probability of extinction under $\mathrm{CM}$ is considerably larger than for EC. In addition, the amount of neutral diversity (heterozygosity, $H$ ) maintained by $\mathrm{CM}$ is lower than that maintained by EC. Although this is just a single and specific example, it illustrates that even if previous purging reduces substantially the inbreeding load of a wild population, a high extinction risk under $\mathrm{CM}$ is not avoided. If a larger reproductive capacity of the species is assumed $(K=2.5)$ under the above scenario of previous purging, extinctions do not occur for either CM or EC management except for $N_{\max }$ as low as 10 , but neutral heterozygosity is lower for CM than for EC (results not shown). There may be some scenarios with low inbreeding load where CM maintains a larger diversity than EC, but the uncertainty about the amount and nature of such a load makes $\mathrm{CM}$ to be a risky method to be applied to species under the risk of extinction.

With regard to the third point raised by $\mathrm{T} \& \mathrm{C} 16$, it is reasonable to assume that some fitness components, such as later life-history traits, traits associated with sexual selection and mating preference are not likely to have a main role in captive breeding populations. However, mutational loads of these components are not included, in general, in the average estimate of $B=6$ of O'Grady et al. (2006), which involves fecundity $(B \approx 2)$, first year survival $(B \approx 1)$ and survival to sexual maturity $(B \approx 3)$.

Finally, regarding the appropriateness of mutational parameters, we agree that the current mutational parameter estimates are uncertain and could be highly variable among species and populations, depending on many factors such as mating system and demographic history. This is why different authors have used different sets of parameter values in their simulations. However, previous simulations on purging selection did not consider $B=6$, although more and more empirical studies suggest even larger values (Hedrick and García-Dorado, 2016). The simulations carried out by Caballero et al. (2016) employed mutational parameters (such as genomic rate, selection and dominance coefficients of mutations) consistent with up-to-date empirical data for mutational parameters as well as with an estimate of $B=6$.

In summary, although there may be a parameter space and some scenarios in which $\mathrm{CM}$ can be effective in reducing inbreeding depression and extinction risk in benign captive conditions, in our
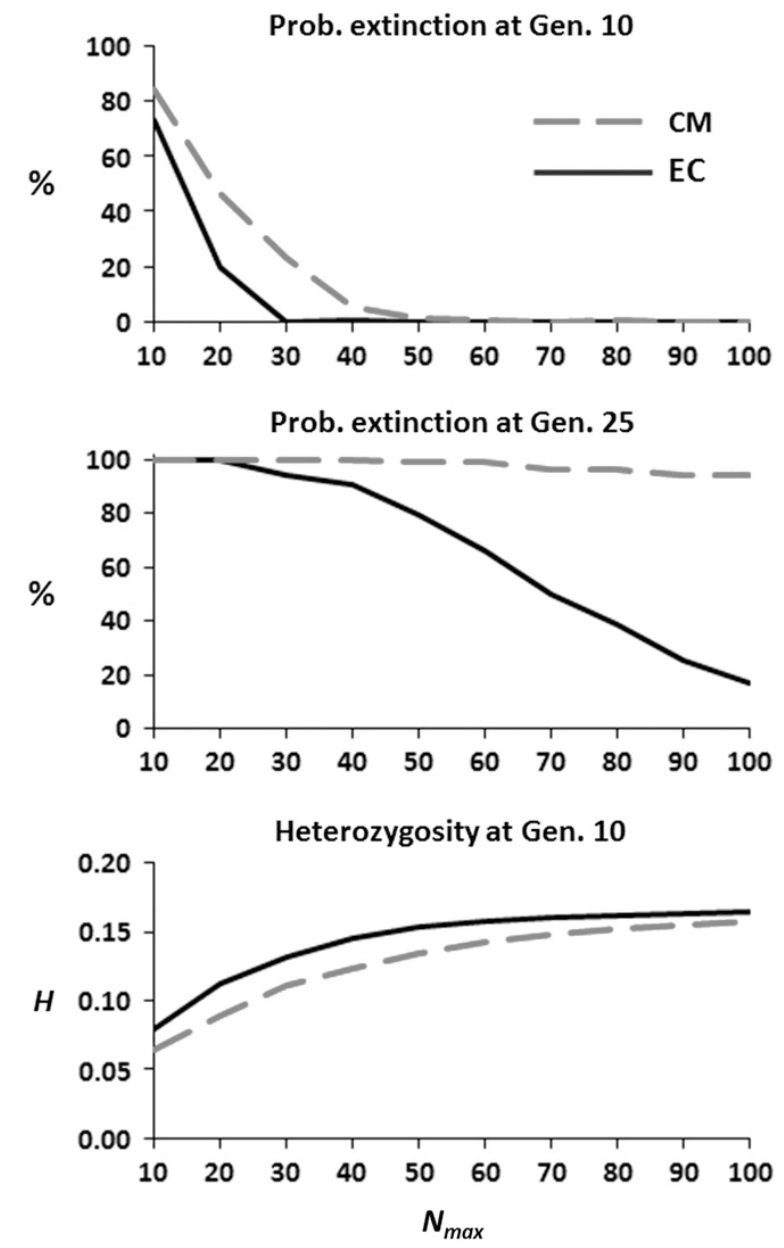

Figure 1 Probability of extinction at generations 10 and 25 (percentage of replicates extinct at that generation) and average expected heterozygosity for neutral genes $(H)$, for populations of maximum size $N_{\max }$ and reproductive rate $K=1.5$ (where $2 K$ is the maximum number of progeny per individual) under mutational model A of Caballero et al. (2016). Previous to the establishment of the captive management, the population of size $N=1000$ individuals suffered a reduction to a size $N=10$ individuals over 20 generations. Selection coefficients of deleterious non-lethal mutations are halved during conservation management. The expected inbreeding depression at the start of the conservation programme corresponds to an inbreeding load of $B=1.2$. Conservation programmes: $\mathrm{CM}$, circular mating; $E C$, equalization of contributions.

opinion, CM should still not be recommended as the best option for managing endangered species in general. This is because an intentional inbreeding programme such as CM for a population kept in benign captive conditions is a double-edged sword. On the one hand, it will reduce the inbreeding load in the benign captive conditions by removing unconditional deleterious mutations of large effect but, on the other hand, the initial higher mortality attached to inbreeding may lead to extinction and/or to an initially lower effective size (see Supplementary Figure S5 of Caballero et al., 2016). This reduction in $N_{e}$ implies a higher fixation of mutations of small deleterious effects or conditional deleterious alleles, possibly rendering the programme harmful rather than useful.

In many inbred line experiments involving various species such as mice and Drosophila, a common observation is that lines go extinct during the process of inbreeding (see, eg, Frankham, 1998, 2005). This experimental setting is similar to purging in captive breeding, both 
being carried out in benign conditions and both being under intentional inbreeding. While experimental populations can afford loss or extinctions, wild and endangered animal species cannot. In many cases, an endangered species may have no populations in the wild and all individuals are in captivity. In such cases, the extinction in captivity means extinction of the species. Thus, CM, or any other intentional inbreeding for the purpose of purging is too risky, especially when many relevant parameters are uncertain. Except when examined and proved experimentally, CM should not be applied to the conservation programmes of any endangered species.

\section{CONFLICT OF INTEREST}

The authors declare no conflict of interest.

\section{ACKNOWLEDGEMENTS}

We thank A García-Dorado, K Theodorou and D Couvet for useful comments. This work was funded by Agencia Estatal de Investigación (AEI) (CGL201675904-C2-1-P), Xunta de Galicia (ED431C 2016-037) and Fondos Feder: 'Unha maneira de facer Europa'.

A Caballero ${ }^{1}$, I Bravo ${ }^{1}$ and $\mathrm{J}$ Wang ${ }^{2}$

${ }^{1}$ Departamento de Bioquímica, Genética e Inmunología, Facultad de Biología, Universidad de Vigo, Vigo, Spain and
${ }^{2}$ Institute of Zoology, Zoological Society of London, London, UK E-mail: armando@uvigo.es

Caballero A, Bravo I, Wang J (2016). Inbreeding load and purging: implications for the short-term survival and the conservation management of small populations. Heredity 118: $177-185$.

Frankham R (1998). Inbreeding and extinction: island populations. Conserv Biol 12: 665-675.

Frankham R (2005). Genetics and extinction. Biol Conserv 126: 131-140.

Fox CW, Reed DH (2011). Inbreeding depression increases with environmental stress: an experimental study and meta-analysis. Evolution 65: 246-258.

Hedrick PW, García-Dorado A (2016). Understanding inbreeding depression, purging, and genetic rescue. Trends Ecol Evol 31: 940-952.

López-Cortegano E, Vilas A, Caballero A, García-Dorado A (2016). Estimation of genetic purging under competitive conditions. Evolution 70: 1856-1870.

Martin G, Lenormand T (2006). The fitness effects of mutations across environments: a survey in the light of fitness landscape models. Evolution 60: 2413-2427.

O'Grady JJ, Brook BW, Reed DH, Ballou JD, Tonkyn DW, Frankham R (2006). Realistic levels of inbreeding depression strongly affect extinction risk in wild populations. Biol Conserv 133: 42-51.

Templeton AR, Read B (1984). Factors eliminating inbreeding depression in a captive herd of Speke's gazelle. Zoo Biol 3: 177-199.

Theodorou K, Couvet D (2015). The efficiency of close inbreeding to reduce genetic adaptation to captivity. Heredity 114: 38-47.

Theodorou K, Couvet D (2017). Circular mating as an option for the genetic management of captive populations: response to Caballero et al. Heredity 119: 49-50. 